\title{
A matemática nos processos de ensino e aprendizagem em física: funções e equações no estudo da quantidade de movimento e sua conservação ${ }^{+*}$
}

\author{
Antonio Jorge Sena dos Anjos ${ }^{1}$ \\ Departamento de Física - Universidade Estadual de Feira de Santana \\ Feira de Santana - BA \\ Concesa Caballero Sahelices ${ }^{2}$ \\ Departamento de Física - Universidad de Burgos \\ Burgos - Espanha \\ Marco Antonio Moreira ${ }^{3}$ \\ Instituto de Física - Universidade Federal do Rio Grande do Sul \\ Porto Alegre - RS
}

\section{Resumo}

O presente estudo trata de uma pesquisa que buscou investigar a relação dialógica entre as aprendizagens significativas de conteúdos da Matemática (funções e equações lineares) e da Física (momento linear e conservação). Ou seja, procurou verificar as possibilidades dos conteúdos matemáticos contribuírem para o aprendizado significativo dos conteúdos da Física e estes para com o aprendizado significativo das funções e equações lineares, no âmbito da Matemática. A Teoria da Aprendizagem Significativa de David Ausubel, juntamente com alguns aportes da Teoria dos Campos Conceituais de Gérard Vergnaud e da Teoria da Mediação de Lev Vygotsky, constituíram-se no referencial teórico desta investigação, tanto para justificativa do problema como para a análise e tratamento dos resultados da investigação. A metodologia de pesquisa teve um enfoque preferencialmente qualitativo, de caráter interpretativo e descritivo, com alguns elementos quantitativos. Os dados foram coletados através de um processo de intervenção didática, constituído por estudos realizados em cinco classes de estudantes de Ensino Médio de distintas instituições do

\footnotetext{
${ }^{+}$Mathematics in teaching and learning processes in physics: functions and equations on the amount of motion study and conservation

* Recebido: novembro de 2016. Aceito: julho de 2017.

${ }^{1}$ E-mail: anjos.antonio@gmail.com, ${ }^{2}$ E-mail: concesa@ubu.es, ${ }^{3}$ E-mail: moreira@if.ufrgs.br
} 
Ensino Médio do Sistema Educacional Brasileiro. Com base na análise dos resultados, concluiu-se que, embora os resultados não tenham sido suficientemente consistentes para um parecer mais abalizado e convincente sobre a possível relação de interferência dialógica entre possíveis aprendizados dos conteúdos matemáticos e físicos, os achados nos conduzem a concluir que os mesmos não são respaldados em considerações de cunhos racional e lógico, a saber: racional, uma vez que razões, como por exemplo, pouco tempo para o processo educativo e complexidade dos campos conceituais e outras já comentadas, não são substanciais para se falar em aprendizagem significativa; lógica, porque não se tendo convicção de aprendizagem dos citados conteúdos, pelas razões já explicitadas, logicamente, não se pode falar de possível interferência recíproca de aprendizados.

Palavras-chave: Ensino de Física; Relações Dialógicas; Aprendizagem Significativa; Funções e Equações Lineares.

\begin{abstract}
The present study aims at investigating the dialogical relation between meaningful learning in Math topics (Functions and Linear Equations) and meaningful learning in Physics (Linear Momentum and Conservation), in an attempt at trying to verify the contribution possibilities of these mathematical topics to the meaningful learning of Physics topics and, moreover, the possibilities of these topics to contribute for the meaningful learning of functions and linear equations in the field of mathematics. The theory of Meaningful Learning by David Ausubel, together with some theoretical points of the Theory of Conceptual Fields by Gérard Vergnaud, and the Mediation Theory by Lev Vygotsky comprised the theoretical basis for this investigation, regarding problem justification, analysis, and treatment of the data collected in this investigation. The research methodology was preferentially based on a qualitative focus of an interpretative and descriptive nature, notwithstanding taking into account some quantitative elements. Data were collected from a teaching intervention process in five classes at different High Schools of the Brazilian Educational System. Based on the analysis of results, conclusions were that although results had not been sufficiently consistent to enable building up a more convincing stand about the likely relation of a dialogical interference between the possible learning of mathematical and physical contents, findings led us to conclude that the learning processes emphasized here were not based
\end{abstract}


on logical or rational considerations. This statement seems feasible since such reasons as lack of time for the development of the educational process to take place and the complexity of the conceptual fields are not substantially enough to evidence the occurrence of meaningful learning; or are not sufficiently logical to convince us that actual learning of the aforementioned contents has happened. Therefore, for reasons already presented, it seems rather difficult to make a clear statement about the existence of a reciprocal interference between mathematical and physical learning processes.

Keywords: Physics Teaching; Dialogical Relations; Meaningful Learning; Functions and Linear Equations.

\section{Introdução}

Entendemos que a Ciência ensinada na Escola deveria ser essencial e fundamental para compreender o mundo de hoje e responder aos seus desafios. Entretanto, a maneira como ela está sendo ensinada, com raríssimas exceções, não atende às demandas exigidas ao educando enquanto estudante e cidadão comum, uma vez que não reúne condições favoráveis para a ocorrência de uma aprendizagem significativa.

Particularmente com a Física, o ensino desenvolvido nas Escolas é tradicionalmente pautado no uso de expressões matemáticas, leis, princípios e conceitos isolados e, sendo assim, a aprendizagem, por consequência, ocorre de forma mecânica, estéril e desvinculada do mundo vivenciado pelo aprendiz, proporcionando-lhe, na maioria das vezes, apenas condições de repetir os enunciados das leis, de memorizar definições e conceitos, além de resolver, com o uso das expressões matemáticas, os problemas propostos nos textos didáticos.

As pesquisas em Ensino de Física (PIETROCOLA, 2002; PINHEIRO et al., 2001; MOREIRA; PINTO, 2003; MOREIRA; KREY, 2006; SHERIN, 2001; WUO, 2000) têm mostrado que, tanto nos livros didáticos quanto nas aulas, os conteúdos de Física são trabalhados com ênfase nas expressões matemáticas, tradicionalmente chamada de “fórmulas", enquanto os conceitos e significados são deixados à margem e descaracterizados no processo histórico e epistemológico de produção do conhecimento. Vale salientar que o entendimento excessivamente matematizado de uma expressão consiste, apenas, em munir o estudante de um instrumento de cálculo, em detrimento do aspecto fenomenológico que ela própria descreve.

Tais constatações induziram-nos a pensar em necessidades de mudanças que privilegiem os conceitos, os significados e a fenomenologia dos conteúdos em detrimento à supervalorização das "fórmulas", sem, entretanto, prescindir delas. Essas mudanças não podem e nem devem ocorrer sem um respaldo teórico e científico, e sim com investigações como esta que possam contribuir cientificamente com elementos para tais mudanças. 
Este trabalho decorre da realização de uma pesquisa científica desenvolvida em duas grandes etapas: a fase exploratória e a pesquisa de campo, nas quais, de alguma forma, buscouse entender o papel da Matemática, no que tange o uso das funções e equações, no ensino de Física, além de verificar as possíveis relações existentes entre essas áreas do saber humano.

$\mathrm{Na}$ fase preliminar, portanto, realizou-se um Estudo Exploratório (ANJOS, 2009) acerca do papel das expressões matemáticas no ensino de Física, com o objetivo de conhecer e avaliar as concepções de estudantes e professores sobre o uso de tais expressões do ponto de vista da Física, além de analisar o tratamento dispensado pelos livros didáticos de Física a essas expressões.

A etapa posterior, a pesquisa de campo, foi desenvolvida em cinco distintas classes de escolas da cidade de Feira de Santana, estado da Bahia, Brasil, tendo como sujeitos, estudantes do Ensino Médio de ambos os sexos, entre 14 e 16 anos. O estudo realizado nesta etapa pretendeu responder ao seguinte problema de pesquisa: Em que medida o aprendizado significativo de funções e equações lineares utilizadas no ensino de determinado conteúdo da Física, pode interferir na aprendizagem significativa desse conteúdo e, reciprocamente, qual a contribuição deste aprendizado para entender significativamente essas expressões?

Assim, a pesquisa foi desenvolvida tendo como objetivo investigar a relação dialética entre as aprendizagens significativas de conteúdos da Matemática e da Física eleitos para realização do estudo. Ou seja, verificar como os estudantes percebem as funções e equações matemáticas nos processos de ensino e aprendizagem da Física e quais as possibilidades dessas expressões matemáticas, quando aprendidas significativamente, contribuírem para a aprendizagem significativa dos conhecimentos físicos e, por outro lado, qual a contribuição da aprendizagem significativa de conteúdos da Física para a aprendizagem das equações e funções.

\section{O estudo exploratório}

Esse estudo foi realizado na cidade de Feira de Santana, estado da Bahia, Brasil, situada a $110 \mathrm{~km}$ de Salvador, capital do estado. O contexto do estudo se constituiu de 03 (três) escolas: Colégio Estadual Hilda Carneiro (Escola Pública), Colégio Gênesis (Escola Privada) e Colégio Padre Ovídio (Escola Privada / Confessional), sendo que em cada uma delas foi usada, para efeito de pesquisa, uma turma de estudantes da segunda série do Ensino Médio. Além destas, muitas outras escolas (públicas e privadas) da cidade complementaram o contexto de realização da pesquisa, uma vez que seus professores foram também investigados.

Os sujeitos deste estudo estão aqui representados por dois grupos distintos de indivíduos. O primeiro grupo compõe-se de 67 (sessenta e sete) estudantes (ambos os sexos) do Ensino Médio do Sistema Educacional Brasileiro, com idades variando entre 14 e 18 anos. O segundo grupo foi composto de 30 professores de Física (ambos os sexos), sendo 23 graduados (12 em Física; 8 em Matemática; 2 em Engenharia Civil; 1 em Mecânica) e 7 graduandos em Física e outras áreas, todos no pleno exercício do magistério em diversas escolas (públicas e privadas) da cidade de Feira de Santana. 
Para efeito de coleta de dados, nesta fase da pesquisa foram usados questionários e entrevistas junto a estudantes e professores, além da análise de conteúdo dos livros didáticos de Física do Programa Nacional do Livro Didático (PNLD)/2008 para o Ensino Médio. Nesses textos do PNLD, foram analisados apenas os capítulos referentes aos conteúdos: "impulso", "quantidade de movimento e sua conservação".

Foram elaborados e utilizados neste estudo dois questionários: um para os estudantes e outro para o professor. Com o questionário para o aluno, com 19 (dezenove) questões abertas, pretendeu-se conhecer o que pensam os estudantes sobre o ensino de Física, particularmente quanto ao significado atribuído por eles às equações matemáticas no estudo de conteúdos da Física. Para o professor, o questionário compreendeu 10 (dez) questões, também versando sobre o ensino de Física, em especial sobre o uso das equações matemáticas nesse processo.

Diante da necessidade de obter mais detalhes para respostas de determinadas questões, foram realizadas entrevistas com os sujeitos da pesquisa. Para os estudantes houve escolha aleatória (ao acaso) e voluntária (respeitando a vontade e predisposição do estudante escolhido) de 2 (dois) alunos de cada turma, enquanto que para os professores, o critério adotado foi levar em consideração os diversos níveis de formação acadêmica: 2 (dois) graduados em Física, 2 (dois) estudantes de Física e 2 (dois) graduados em outras áreas distintas da Física.

Considerando as análises e resultados, aliados às teorias que dão suporte ao estudo, concluiu-se com esta fase da pesquisa que as equações matemáticas desempenham um papel de fundamental importância no ensino da Física, em particular no processo de conceitualização, por se constituírem em representações simbólicas usadas pelos estudantes para dar significado aos conceitos e, assim sendo, a sua utilização deveria ser objeto de atenção para quem as usam, tanto na exposição dos conteúdos de Física nos textos didáticos, como no planejamento e execução de sequências didáticas desenvolvidas junto aos alunos em atividades de estudo.

(Re) significar o papel das equações matemáticas no ensino de Física, através de propostas metodológicas alternativas que atentem para a necessidade de se promover uma aprendizagem significativa dos conteúdos, foi o que nos propusemos a fazer com base neste estudo.

Nesse sentido, investimos em avançar na busca por uma aprendizagem significativa de conteúdos da Física (quantidade de movimento e sua conservação) e da Matemática (funções e equações lineares). Para tanto, prosseguimos o trabalho de investigação, agora em sala de aula, na tentativa de validar propostas de intervenção didática, tendo como referência a Teoria da Aprendizagem Significativa (TAS) de Ausubel e alguns elementos da Teoria dos Campos Conceituais (TCC) de Vergnaud, permeada pela ideia de Mediação de Vygotsky, tendo em vista o processo de construção do conhecimento como uma interação mediada por várias relações.

\section{Revisão de literatura}

Com o propósito de mapear o que vem sendo pesquisado na área, possibilitando ao pesquisador ter uma visão geral do estágio de desenvolvimento das pesquisas sobre os vínculos 
entre a Matemática e a Física e, mais particularmente, sobre o papel da matemática nos processos de ensino e de aprendizagem em Física, foram consultados e revisados trabalhos de pesquisa, em diversos e renomados periódicos e eventos (nacionais e internacionais) de investigação em ensino de Ciências, ensino de Física e em educação Matemática, no período compreendido entre os anos 2000 e 2014.

Certamente, a revisão desses trabalhos forneceu elementos que cooperaram para justificar a importância desta investigação, além de permitir situar a pesquisa no contexto acadêmico e contribuir para análise dos resultados e argumentações futuras. Tanto no aspecto históricoepistemológico como no didático-pedagógico, o uso da Matemática na estruturação do conhecimento físico é uma constatação, é um fato, é uma realidade.

Após análise, os trabalhos foram agrupados em categorias, conforme o enfoque de cada pesquisa, tais como, investigações sobre: 1) Dificuldades de aprendizagem; 2) Estratégias de ensino; 3) Concepções e raciocínio; 4) Contexto histórico-epistemológico; 5) Comportamento cognitivo-epistemológico. No Quadro 1, apresenta-se um resumo geral com a caracterização dos trabalhos analisados para efeito da revisão de literatura, conforme classificação por categoria de análise.

Das obras analisadas, podemos concluir que, sejam na perspectiva do ensino ou da aprendizagem, os trabalhados selecionados e que aqui compõem a revisão de literatura da investigação, revestem-se de um propósito básico que é o de entender e buscar ressignificar o papel da Matemática no ensino da Física.

Quaisquer que sejam as perspectivas das investidas de pesquisas, todas elas têm em comum a preocupação precípua de colocar a Matemática no seu devido lugar no ensino das Ciências, particularmente no ensino da Física, visando obter, através de uma relação harmônica entre estas duas áreas do saber, bons resultados para a prática didático-pedagógica de ambas. Apesar de reconhecer as diferenças existentes entre o fazer matemático, que não necessita de vínculo imediato com a realidade e o fazer físico, que se utiliza de modelos matemáticos para representar sua realidade, é fundamental e essencial a busca pela integração entre estas áreas no contexto da Escola (KARAM, 2004).

Portanto, é de vital importância a compreensão do papel da Matemática na construção dos conhecimentos físicos, como também é importante saber usá-la adequadamente no estudo e resolução de situações problemáticas na Física como linguagem de poder preditivo e estruturante do conhecimento físico.

Por seu turno, o conhecimento sobre a natureza do saber físico se constitui numa das variáveis que os professores precisam considerar na elaboração e na implementação de intervenções didático-pedagógicas na perspectiva de uma aprendizagem significativa, como se propõe nesta investigação.

Assim, também, e não menos importante, é o conhecimento histórico-epistemológico das relações entre a Física e a Matemática e o reflexo deste conhecimento no comportamento cognitivo do educando. 
Outro aspecto levantado nos estudos revisados é a necessária sintonia entre os processos de ensino e aprendizagem, os aspectos como contextualização e interdisciplinaridade, considerando a Matemática como linguagem estruturante do pensamento físico e, por consequência, de forte influência no comportamento cognitivo dos aprendizes na construção de conhecimentos físicos.

Quadro 1: Caracterização dos trabalhos utilizados na revisão de literatura.

\begin{tabular}{|c|c|c|c|}
\hline $\begin{array}{l}\text { CATEGO- } \\
\text { RIA }\end{array}$ & TÍTULO & AUTOR & FONTE \\
\hline \multirow{4}{*}{$\begin{array}{l}\text { 1.1. Difi- } \\
\text { culdades } \\
\text { de aprendi- } \\
\text { zagem } \\
\text { (04 traba- } \\
\text { lhos) }\end{array}$} & $\begin{array}{l}\text { Análisis de la utilización y comprensión del } \\
\text { Cálculo Diferencial en la enseñanza de la Física }\end{array}$ & $\begin{array}{l}\text { López-Gay, R.,Martínez } \\
\text { Torregrosa, J., Gras } \\
\text { Martí, A. }\end{array}$ & $\begin{array}{l}\text { Enseñanza de las Ci- } \\
\text { enciais, 2005, 23(3), } \\
\text { 321-334 }\end{array}$ \\
\hline & $\begin{array}{l}\text { Dificuldades dos alunos na aprendizagem da Lei } \\
\text { de Gauss em nível de Física Geral à luz da Teo- } \\
\text { ria dos Modelos Mentais de Johson-Laird }\end{array}$ & Moreira, M. A., Krey, I. & $\begin{array}{l}\text { Revista Brasileira de } \\
\text { Ensino de Física, } \\
\text { 2006, 28(3), 353-360 }\end{array}$ \\
\hline & $\begin{array}{l}\text { Dificuldades dos alunos na aprendizagem da Lei } \\
\text { de Ampére, à luz da Teoria dos Modelos Mentais } \\
\text { de Johson-Laird }\end{array}$ & $\begin{array}{l}\text { Moreira, M. A. e Pinto, } \\
\text { A. O. }\end{array}$ & $\begin{array}{l}\text { Revista Brasileira de } \\
\text { Ensino de Física, } \\
\text { 2003, 25(3), 317-324 }\end{array}$ \\
\hline & $\begin{array}{l}\text { Dificultades en la aplicación del cálculo diferen- } \\
\text { cial e integral en la resolución de problemas del } \\
\text { trabajo efectuado por un gas ideal: Conceptos y } \\
\text { teoremas en acción }\end{array}$ & Alvarado, M. S. A. & $\begin{array}{l}\text { Latin American Jour- } \\
\text { nal of Physics Educa- } \\
\text { tion, } 2012,6(1) \text {, pp. } \\
106-115 \text {. }\end{array}$ \\
\hline \multirow{8}{*}{$\begin{array}{l}\text { 1.2. Estra- } \\
\text { tégias de } \\
\text { ensino } \\
(08 \text { traba- } \\
\text { lhos })\end{array}$} & Enseñanza de equaciones lineales en contexto & $\begin{array}{l}\text { Galagovsky, L.R. Y Cit- } \\
\text { tadini,P.E. }\end{array}$ & $\begin{array}{l}\text { Enseñanza de las Ci- } \\
\text { enciais, 2008, } 26 \text { (3), } \\
\text { 359-374. }\end{array}$ \\
\hline & $\begin{array}{l}\text { How to Promote the Learning of Physics from } \\
\text { Formulae? }\end{array}$ & $\begin{array}{l}\text { Bagno,E.; Eylon,B. and } \\
\text { Berger, H. }\end{array}$ & $\begin{array}{l}\text { GIREP-EPEC SYM- } \\
\text { POSIUM, } 2009\end{array}$ \\
\hline & $\begin{array}{l}\text { O conceito de função e sua linguagem para os } \\
\text { professores de Matemática e de Ciências }\end{array}$ & $\begin{array}{l}\text { Zuffi, E. M. e Pacca, J. L. } \\
\text { de A. }\end{array}$ & $\begin{array}{l}\text { Ciência e Educação, } \\
\text { 2002,8(1),1-12 }\end{array}$ \\
\hline & $\begin{array}{l}\text { O conhecimento físico e sua relação com a Ma- } \\
\text { temática: um olhar voltado para o ensino médio }\end{array}$ & Martini, M. da G. de A. & $\begin{array}{l}\text { Universidade de São } \\
\text { Paulo (USP), São } \\
\text { Paulo-SP, Brasil, } \\
\text { 2006, 151p. }\end{array}$ \\
\hline & $\begin{array}{l}\text { Views about Physics held by Physics teachers } \\
\text { with differing approaches to teaching Physics }\end{array}$ & $\begin{array}{l}\text { Mulhall, P. and Gun- } \\
\text { stone, R. }\end{array}$ & $\begin{array}{l}\text { Research Science } \\
\text { Education, 2008, V. } \\
38,435-462\end{array}$ \\
\hline & $\begin{array}{l}\text { Fragmentações e aproximações entre Matemá- } \\
\text { tica e Física no contexto escolar: problemati- } \\
\text { zando o conceito de função afim }\end{array}$ & Lopes, J. P. & $\begin{array}{l}\text { Universidade Federal } \\
\text { de Santa Catarina, } \\
\text { Florianópolis (SC), } \\
\text { Brasil, 2004, 205 p. }\end{array}$ \\
\hline & $\begin{array}{l}\text { Sobre la aplicación y uso del Concepto de Deri- } \\
\text { vada en el Estudio de Conceptos Económicos en } \\
\text { Estudiantes de Bachillerato y Universidad }\end{array}$ & Ariza, Á. y Llinares, S. & $\begin{array}{l}\text { Enseñanza de las Ci- } \\
\text { encias, 2009, 27(1), } \\
121-136 .\end{array}$ \\
\hline & $\begin{array}{l}\text { O Cálculo nas aulas de Física da UFRGS: Um } \\
\text { Estudo Exploratório }\end{array}$ & $\begin{array}{l}\text { Santarosa, M. C. P. e } \\
\text { Moreira, M. A. }\end{array}$ & $\begin{array}{l}\text { Investigações em En- } \\
\text { ino de Ciências, } \\
2011,16(2) \text {, pp. } 317- \\
351 .\end{array}$ \\
\hline \multirow{2}{*}{$\begin{array}{l}\text { 1.3. Con- } \\
\text { cepções e } \\
\text { raciocínio } \\
\text { (06 traba- } \\
\text { lhos) }\end{array}$} & How students understand physics equations & Shering, B. I. & $\begin{array}{l}\text { Cognition and In- } \\
\text { struction, 2001, } \\
\text { 19(4), 479-541 }\end{array}$ \\
\hline & $\begin{array}{l}\text { Mathematics in Physics Education: scaning his- } \\
\text { torical evolution of the differencial to find a } \\
\text { more appropriate model for teaching Differen- } \\
\text { cial Calculus in Physics }\end{array}$ & $\begin{array}{l}\text { Martinez-Torregrosa, J.; } \\
\text { Lopez-Gay, R. and Gras- } \\
\text { Marti, A. }\end{array}$ & $\begin{array}{l}\text { Science and Educa- } \\
\text { tion, 2006, } 15 \text { (5), } \\
447-462 .\end{array}$ \\
\hline
\end{tabular}




\begin{tabular}{|c|c|c|c|}
\hline $\begin{array}{c}\text { CATEGO- } \\
\text { RIA }\end{array}$ & TÍTULO & AUTOR & FONTE \\
\hline & $\begin{array}{l}\text { Que hácen y qué entienden los estudiantes y pro- } \\
\text { fesores de Física cuando usan expressiones dife- } \\
\text { renciales? }\end{array}$ & $\begin{array}{l}\text { López-Gay, R. y Marti- } \\
\text { nez Torregrosa, J. }\end{array}$ & $\begin{array}{l}\text { Enseñanza de las } \\
\text { Cienciais, 2005, } 23 \\
\text { (3), 331-334. }\end{array}$ \\
\hline & What makes physics difficult? & $\begin{array}{l}\text { Ornek, F.; Robinson, W. } \\
\text { R. and Mark P. Haugan, } \\
\text { M. P. }\end{array}$ & $\begin{array}{l}\text { International Journal } \\
\text { of Environmental \& } \\
\text { Science Education, } \\
2008,3(1), 30-34\end{array}$ \\
\hline & $\begin{array}{l}\text { What does ' } \mathrm{i} \text { understand the equation' really } \\
\text { mean? }\end{array}$ & Hechter, R. P. & $\begin{array}{l}\text { Physics Education, } \\
2010,45(2), 132-133 .\end{array}$ \\
\hline & $\begin{array}{l}\text { Coordination of Mathematics and Physical Re- } \\
\text { sources by Physics Graduate Students }\end{array}$ & $\begin{array}{l}\text { Ayush, G., Redish, E. F. } \\
\text { \& Hammer, D. }\end{array}$ & $\begin{array}{l}\text { AIP Conference Pro- } \\
\text { ceedings, 2007, Vol. } \\
\text { 951, pp. 104-107. }\end{array}$ \\
\hline \multirow{5}{*}{$\begin{array}{l}\text { 1.4. Con- } \\
\text { texto histó- } \\
\text { rico-episte- } \\
\text { mológico } \\
\text { (05 traba- } \\
\text { hos) }\end{array}$} & $\begin{array}{l}\text { Fórmulas e palavras: reflexões sobre o ensino da } \\
\text { Lei de Coulomb }\end{array}$ & Oliveira, A. T. de S. & $\begin{array}{l}\text { Universidade Federal } \\
\text { Fluminense, Rio de } \\
\text { Taneiro, Brasil, 2003, } \\
\text { 135p. }\end{array}$ \\
\hline & $\begin{array}{l}\text { Habilidades técnicas versus habilidades estrutu- } \\
\text { rantes: resolução de problemas e o papel da Ma- } \\
\text { temática como estruturante do pensamento cien- } \\
\text { tífico. }\end{array}$ & $\begin{array}{l}\text { Karam, R. e Pietrocola, } \\
\text { M. }\end{array}$ & $\begin{array}{l}\text { ALEXANDRIA Re- } \\
\text { vista de Educação em } \\
\text { Ciência e Tecnologia, } \\
\text { 2009, } 2 \text { (2),181-205 }\end{array}$ \\
\hline & $\begin{array}{l}\text { Recognizing the structural role of mathematics } \\
\text { in physical thought }\end{array}$ & $\begin{array}{l}\text { Karam, R. e Pietrocola, } \\
\text { M. }\end{array}$ & $\begin{array}{l}\text { European Science } \\
\text { Education Research } \\
\text { Association Confer- } \\
\text { ence. Istanbul, Tur- } \\
\text { key, 2009, 1-10 p.p. }\end{array}$ \\
\hline & $\begin{array}{l}\text { Science and Mathematics: a relationship in need } \\
\text { of counseling? }\end{array}$ & Orton, T. and Roper, T. & $\begin{array}{l}\text { Studies in Science } \\
\text { Education, 2000, 35, } \\
\text { 123-154 pp. }\end{array}$ \\
\hline & $\begin{array}{l}\text { A matemática como estruturante do conheci- } \\
\text { mento físico }\end{array}$ & Pietrocola, M. & $\begin{array}{l}\text { Caderno Brasileiro de } \\
\text { Ensino de Física, } \\
2002,19(1), 93-114 .\end{array}$ \\
\hline \multirow{6}{*}{$\begin{array}{l}\text { 1.5. Com- } \\
\text { portamento } \\
\text { cognitivo- } \\
\text { epistemo- } \\
\text { lógico } \\
\text { (06 traba- } \\
\text { lhos) }\end{array}$} & $\begin{array}{l}\text { Problem Solving and the use of Math in Physics } \\
\text { Courses }\end{array}$ & Redish, E. F., & $\begin{array}{l}\text { World View on Phys- } \\
\text { ics Education in } \\
\text { 2005: Focusing on } \\
\text { Change }\end{array}$ \\
\hline & $\begin{array}{l}\text { Using Math in Physics: Warrants a Epistemolog- } \\
\text { ical Frames }\end{array}$ & Edward F. Redish, E. & $\begin{array}{l}\text { GIREP-EPEC Sym- } \\
\text { posium, } 2009\end{array}$ \\
\hline & $\begin{array}{l}\text { An exploration of university physics students' } \\
\text { epistemological mindsets towards the under- } \\
\text { standing of physics equations }\end{array}$ & $\begin{array}{l}\text { Domert, D.; Airey, J.; } \\
\text { Kung, R. and Linder, C. }\end{array}$ & $\begin{array}{l}\text { NorDiNa-Nordic } \\
\text { Studies in Science } \\
\text { Education, 2007, } 3 \\
\text { (1),15-28. }\end{array}$ \\
\hline & $\begin{array}{l}\text { Estudo exploratório sobre as relações entre co- } \\
\text { nhecimento conceitual, domínio de técnicas ma- } \\
\text { temáticas e resolução de problemas em estudan- } \\
\text { tes de licenciatura em Física. }\end{array}$ & $\begin{array}{l}\text { Ataíde, A. R. P. e Greca, } \\
\text { I. M. }\end{array}$ & $\begin{array}{l}\text { Revista Eletrónica de } \\
\text { Enseñanza de las } \\
\text { Ciencias, 2013, } 12 \\
\text { (1), 209-233. } \\
\end{array}$ \\
\hline & On the Role of Mathematics in Physics & Quale, A. & $\begin{array}{l}\text { Science \& Education, } \\
2011 \text {, V. 20, pp. 359- } \\
372\end{array}$ \\
\hline & $\begin{array}{l}\text { On the Role of Mathematics in Physics: A Con- } \\
\text { structivist Epistemic Perspective }\end{array}$ & Quale, A. & $\begin{array}{l}\text { Science \& Education, } \\
2011, \text { V. } 20 \text {, pp. } 609- \\
624\end{array}$ \\
\hline
\end{tabular}


Em geral, as investigações aqui selecionadas para efeito dessa revisão de literatura do trabalho, embora admitam a inexistência de uma resposta simples para a organização e elaboração de aulas de Matemática que garantam o aprendizado do saber físico, não sinalizam com possibilidades concretas de um fazer didático-pedagógico nos campos dos saberes físicos e matemáticos, bem como a relação dialética entre eles tendo em vista a possibilidade de uma aprendizagem significativa.

Nesse sentido, este estudo pretendeu contribuir para suprir esta lacuna, uma vez que buscou não só investigar possibilidades de ocorrência de aprendizagem significativa nos campos da Física e da Matemática como também tratou de verificar em que medida estes aprendizados se interferem mutuamente.

\section{Marco teórico}

Para efeito de desenvolvimento do estudo e análise dos seus resultados, adotou-se um referencial teórico que aqui se apresenta sob dois pontos de vista a saber: da Psicologia Cognitiva; das Ciências Física e Matemática.

Da Psicologia Cognitiva, adotou-se como referencial teórico, basicamente, a Teoria da Aprendizagem Significativa (AUSUBEL, 2003). Além desta, quando necessário, fez-se uso de alguns aportes da Teoria da Mediação (VYGOTSKY, 1995) e da Teoria dos Campos Conceituais (VERGNAUD, 1993) que, em momentos oportunos, foram devidamente utilizadas.

A Teoria da Aprendizagem Significativa (TAS) tem como foco principal a aprendizagem cognitiva que, segundo o próprio Ausubel,

[...] por definição envolve a aquisição de novos significados. Estes são, por sua vez, os produtos finais da aprendizagem significativa. Ou seja, o surgimento de novos significados no aprendiz reflete a ação e a finalização anteriores no processo de aprendizagem significativa (Ausubel, 2003, p. 71).

Ausubel tem sua atenção sempre voltada para a aprendizagem que ocorre no cotidiano das Escolas. Ele considera aquilo que o estudante já sabe como sendo o fator que mais influencia no seu aprendizado. Na sua teoria, o conceito central é o conceito de aprendizagem significativa. Segundo Moreira (2006),

[...] a aprendizagem significativa é um processo por meio do qual uma nova informação se relaciona, de maneira substantiva (não literal) e não arbitrária, a um aspecto relevante da estrutura cognitiva do indivíduo. Neste processo a nova informação interage com uma estrutura de conhecimento específica, a qual Ausubel chama de 'conceito subsunçor' ou, simplesmente, 'subsunçor', existente na estrutura cognitiva de quem aprende (p. 15).

Observemos, entretanto, que na TAS fala-se que a nova informação relaciona-se de forma "não literal" e "não arbitrária", com aspectos relevantes da estrutura cognitiva, o que 
não significa, portanto, não se tratar de uma aprendizagem mecânica, na qual as novas informações pouco ou nada interagem com os conceitos relevantes e pré-existentes na mente do indivíduo, mas sim um processo que ofereça condições para que a aprendizagem ocorra significativamente.

Do ponto de vista das Ciências Física e Matemática, o campo conceitual da Mecânica Clássica se constituiu em objeto de interesse da Física, tendo em vista o fato do conteúdo eleito para esta investigação concentrar-se nesta área e que tem a sua origem nas ideias de Galileu, cuja obra contém as principais bases sobre as quais se assentou a Física newtoniana.

Com uma relação bastante estreita com o conteúdo da Física (momento linear) eleito para efeito da investigação, os tópicos de funções e equações lineares, surgem naturalmente pela sua íntima relação com o primeiro e, como tal, credenciam-se como os referentes teóricos do ponto de vista da Matemática para o desenvolvimento deste estudo.

\section{Marco metodológico}

Para realização deste trabalho optou-se pelo estudo de caso etnográfico, com base nos resultados advindos da pesquisa exploratória (Anjos, 2009), a qual previu a utilização de alguns métodos e técnicas de coleta de dados com finalidades distintas, porém coerentes com o referencial metodológico adotado para este estudo. Trata-se de um estudo de caso, uma vez que este procura enfatizar o conhecimento do singular e, nessa investigação, a instância singular foi cada classe de estudantes do primeiro/segundo ano do Ensino Médio que fez parte da pesquisa. Ou seja, cada uma dessas classes (grupo distinto de indivíduos) foi submetida a uma proposta metodológica de ensino, se constituindo num caso particular de um estudo desenvolvido durante uma unidade letiva.

Conforme Ludke e André (2003, p.18-20), os estudos de caso justificam-se para esse trabalho uma vez que: "visam a descoberta"; "enfatizam a interpretação em contexto"; "buscam retratar a realidade de forma completa e profunda"; "usam uma variedade de fontes de informação"; "revelam uma experiência vicária e permitem generalizações naturalísticas"; "procuram representar os diferentes e às vezes conflitantes pontos de vista"; além de fazerem "uso de uma linguagem e uma forma mais acessível que os outros relatórios de pesquisa". Algumas dessas características mencionadas não só estão em sintonia com a natureza da investigação, como definem e classificam o tipo de estudo de caso desse trabalho. Um estudo de dupla natureza: descritiva e interpretativa.

É também um estudo etnográfico, pois se trata de uma adaptação da etnografia à educação, onde a preocupação do pesquisador é com o processo educativo de um grupo social. A etnografia, dita tradicional, usada pelos antropólogos e sociólogos para o estudo da cultura de um grupo social, começou a ser usada pelos pesquisadores em educação (Ludke e André, 2003), que com aproximações e adaptações incorporaram-na como uma nova linha de pesquisa denominada "antropológica" ou "etnográfica". 
Para efeito de coleta de dados, fez-se uso de alguns instrumentos como: Testes de Sondagem, Intervenções Didáticas, Observações, Teste de Avaliação Final (TAF) e Entrevistas, sobre os quais faremos uma breve descrição conforme suas finalidades e pertinência de utilização.

Com a finalidade precípua de conhecer as preconcepções dos sujeitos da pesquisa, foi aplicado junto às classes de estudantes um Teste de Sondagem em Matemática (TSM) e um Teste de Sondagem em Física (TSF). Ambos os testes objetivaram não apenas conhecer as concepções prévias dos sujeitos em Matemática (funções e equações lineares) e Física (quantidade de movimento linear e sua conservação), respectivamente, mas, também, tendo conhecimento da sua existência, buscar tratá-las devidamente sob o ponto de vista do ensino, com a elaboração e implementação de uma Unidade de Didática de Ensino (UDE), visando à construção de novos conhecimentos e da sua aprendizagem significativa.

Além da participação e avaliação de especialistas nas áreas de Física e Matemática, o TSM e o TSF apresentaram alfas de Cronbach iguais a 0,93 e 0,66, respectivamente, que atestam a fidedignidade destes instrumentos.

As Intervenções Didáticas (ID), em número de 8 (oito), compuseram uma UDE visando oportunizar a realização de distintas atividades de ensino, desenvolvendo estudos sobre os conteúdos de Matemática e de Física envolvidos no estudo, objetivando favorecer a ocorrência da aprendizagem significativa dos conteúdos, ao tempo em que se propunha verificar a relação dialética entre estas aprendizagens, além de contribuir para coleta de dados, através de Observações livres e registros do professor/pesquisador, bem como das informações e produções dos estudantes oriundas das diversas atividades (leituras e discussão de textos, experimentos, simulações, vídeos, situações-problema, exercícios,...) desenvolvidas ao longo das intervenções.

Ao final da realização do conjunto das intervenções didáticas, foi aplicado o TAF (Alfa de Cronbach $=0,85$ ), composto de 6 (seis) questões, com o objetivo de verificar o nível de aprendizagem dos sujeitos referente aos conteúdos de Matemática e Física, decorrente da implementação da UDE.

Com a finalidade de detalhar e ampliar o espectro das informações já obtidas nas fases anteriores de coleta de dados, utilizou-se o recurso da Entrevista, contendo novos questionamentos formulados a partir de respostas já conhecidas, oriundas do TAF e de alguns registros nas intervenções (ID).

Para efeito de análise dos resultados e consonantes com o problema de pesquisa do trabalho, se fez necessário obter informações para além do nível de aprendizagem individual do estudante, ou seja, de como ocorreu o progresso dessa aprendizagem relativo aos conteúdos da Matemática (funções e equações lineares) e da Física (quantidade de movimento e sua conservação). Para tanto, buscou-se fazer uso de parâmetros que permitissem identificar em que medida esse desempenho ocorrera, aos quais se denominou Indicadores de Progressividade da Aprendizagem (IPA) 
Esses indicadores (IPA), definidos e valorados a priori, estiveram associados às competências dos estudantes em cada um dos conteúdos estudados, conforme o planejamento da UDE, e, do ponto de vista da pesquisa, foram verificados junto aos instrumentos de registros individuais de dados (TSM, TSF, TAF). Como tal, os IPA serviram de parâmetros norteadores para o procedimento interpretativo, visando identificar indícios que poderiam contribuir para facilitar os aspectos conclusivos desse estudo.

\section{Resultados}

Cada um dos Estudos realizados ocorreu com base na implementação de uma Unidade Didática de Ensino (UDE), composta de um conjunto de Intervenções Didáticas (ID) que, aliadas aos instrumentos e/ou expedientes de coleta de dados se buscou, de alguma maneira, encontrar elementos que, em potencial, poderiam contribuir para responder a questão de pesquisa.

Vale ressaltar o importante papel da UDE, tanto na sua precípua função de promover e facilitar o processo de ensino com vistas à aprendizagem significativa dos conteúdos trabalhados, como na condição de expediente que possibilitou a realização do processo investigativo, assegurando, no espaço das intervenções didáticas, condições efetivas para que a coleta de dados pudesse ser ampliada, através das observações e registros feitos ao longo do desenvolvimento das atividades em cada estudo.

Visando integralizar os estudos realizados, foram propostos alguns questionamentos com a finalidade de contribuir para as considerações conclusivas deste trabalho: Quais os resultados revelados em cada um dos Estudos? Quais as regularidades observadas nas contribuições de cada Estudo? Os resultados obtidos contribuíram suficientemente para responder a questão de pesquisa? Tendo em vista os resultados obtidos nos distintos Estudos, o que se pode concluir acerca do papel do conhecimento matemático na construção de saberes em Física no Ensino Médio?

Quadro 2: Sínteses dos resultados decorrentes das investigações realizadas em cada Estudo.

\begin{tabular}{|l|l|}
\hline ESTUDOS & \multicolumn{1}{|c|}{ SÍNTESES DOS RESULTADOS } \\
\hline Estudo I & O TAF, juntamente com TSM e TSF e as ID, proporcionaram-nos uma ideia geral do desem- \\
(Estudo & penho da UDE, apontando para as necessidades de possíveis adequações nos estudos futuros: \\
Piloto) & $\begin{array}{l}\text { 1) trabalhar previamente, quando necessário, os conteúdos relacionados com vetores e leis de } \\
\text { Newton; 2) melhorar o processo de mediação junto aos grupos, nos momentos de discussão } \\
\end{array}$ \\
& $\begin{array}{l}\text { interna e no compartilhamento dos construtos com toda classe; 3) reestruturar o TAF, tendo em } \\
\text { vista algumas lacunas e fragilidades apresentadas. }\end{array}$ \\
\hline
\end{tabular}




\begin{tabular}{|c|c|}
\hline Estudo II & $\begin{array}{l}\text { Os estudantes esboçaram possuir domínio da relação entre as variáveis de uma função linear, } \\
\text { particularmente quantidade de movimento }(\mathbf{Q}) \text { e velocidade }(\mathbf{v}) \text { e demonstraram também obter } \\
\text { conhecimentos para operar com as equações. } \\
\text { Observou-se, nas atividades relacionadas ao princípio de conservação da quantidade de movi- } \\
\text { mento, certo domínio quanto ao uso das equações matemáticas, não apenas pelos índices cres- } \\
\text { centes das médias atribuídas aos IPA6 e IPA7, mas também pelo que foi revelado nas suas } \\
\text { produções dos estudantes e explicitado nas entrevistas, que podem ser traduzidos como papel } \\
\text { estruturante da matemática no conhecimento físico. }\end{array}$ \\
\hline Estudo III & $\begin{array}{l}\text { Os indícios detectados neste estudo: } 1 \text { ) À medida que se avançou no processo instrucional, } \\
\text { com a mediação docente e de conteúdos, e as oportunidades de trocas nos grupos, notou-se uma } \\
\text { considerável evolução no aprendizado sobre funções lineares como se observa nas interven- } \\
\text { ções, particularmente na ID7; 2) ficou evidenciado, nas etapas que envolveram exercícios e } \\
\text { situações problemáticas no contexto da Física, que o conhecimento sobre as equações foi de } \\
\text { fundamental importância para resolução destas atividades, não apenas como domínio técnico, } \\
\text { mas, talvez, como "habilidade estruturante"; 3) as propostas de resolução no TAF e as respostas } \\
\text { concedidas por conta das entrevistas confirmam o desempenho dos estudantes no que tange à } \\
\text { aprendizagem do princípio de conservação da quantidade de movimento, revelando que a mai- } \\
\text { oria dos indivíduos não conseguiu responder as questões relativas a este tópico da Física. }\end{array}$ \\
\hline Estuc & $\begin{array}{l}\text { Os indícios constatados revelaram que: } 1 \text { ) os estudantes demonstraram conhecimentos para } \\
\text { operar com as equações lineares, o que não ocorreu com a mesma intensidade com funções } \\
\text { lineares; 2) o conceito de quantidade de movimento foi aprendido mais sob o aspecto da repre- } \\
\text { sentação matemática do que da Física e, quanto ao princípio de conservação do momento linear, } \\
\text { a maioria dos alunos demonstrou não possuir conhecimentos para tratar com situações proble- } \\
\text { máticas; 3) foi percebida a existência de uma estreita relação do aprendizado sobre as equações } \\
\text { lineares com o seu trato no contexto da Física. }\end{array}$ \\
\hline Estudo V & $\begin{array}{l}\text { Os indícios constatados nos levaram a inferir que: } 1 \text { ) A maioria dos estudantes demonstrou } \\
\text { ter adquirido conhecimentos conceituais e procedimentais para operar com as equações lineares } \\
\text { e entender funções lineares; 2) Percebeu-se que o conceito de quantidade de movimento foi } \\
\text { apreendido na forma da representação matemática, deixando à margem a conceituação física; } \\
\text { 3) Os estudantes demonstraram não dominar conceitos e procedimentos sobre o princípio de } \\
\text { conservação da quantidade de movimento; 4) foi constatada a existência de uma relação de } \\
\text { contribuição do contexto da Física no aprendizado das funções e equações lineares. Entretanto, } \\
\text { verificou-se que o mesmo não ocorrera no sentido inverso. }\end{array}$ \\
\hline
\end{tabular}

As respostas a tais indagações são facilitadas na medida em que se traz para reflexão os resultados de cada Estudo e apresentados nos quadros 2 e 3. Os elementos componentes do Quadro 2 configuram-se como sendo indícios e/ou regularidades e, portanto, resultados revelados no processo investigativo de cada caso (Estudo) que, por sua vez, constituem-se em elementos vitais para efeito de elaboração das considerações conclusivas de cada Estudo.

Quanto às considerações conclusivas, elas se encontram devidamente apresentadas no Quadro 3 e, como tal, possibilitam uma visão mais crítica, reflexiva e integradora dos Estudos, 
além de apresentar elementos que colaboraram para responder as demais indagações formuladas, contribuindo, dessa forma, para a conclusão do trabalho. Diante desses resultados e de suas análises, buscou-se responder a seguinte indagação: Os resultados obtidos contribuíram suficientemente para responder a questão de pesquisa? Neste questionamento, se observa basicamente duas premissas fundamentais e distintas. A primeira diz respeito à aprendizagem significativa dos conteúdos matemáticos e físicos, enquanto a segunda refere-se à relação de interferência dialógica entre estes conhecimentos.

Quadro 3: Sínteses das considerações conclusivas decorrentes das análises dos resultados.

\begin{tabular}{|c|c|}
\hline ESTUDOS & SÍNTESES DAS CONSIDERAÇÕES CONCLUSIVAS \\
\hline $\begin{array}{l}\text { Estudo I } \\
\text { (Estudo Pi- } \\
\text { loto) }\end{array}$ & $\begin{array}{l}\text { O Estudo I atingiu seus objetivos, na medida em que cumpriu com aquilo a que se propôs, ou } \\
\text { seja, testou e validou na prática o protocolo de uma Unidade Didática de Ensino, tanto no as- } \\
\text { pecto pedagógico dos processos de ensino e aprendizagem, quanto no aspecto da investigação, } \\
\text { oportunizando a coleta de dados, com vistas a responder a questão de pesquisa. }\end{array}$ \\
\hline Estudo II & $\begin{array}{l}\text { Com base nas análises dos resultados, nas revelações dos IPA e nos indícios apresentados, con- } \\
\text { cluímos que o Estudo II reuniu elementos que, aparentemente, sinalizaram para a existência de } \\
\text { uma possível relação de implicação dialógica entre os aprendizados dos conhecimentos físicos } \\
\text { e matemáticos, limitando-se, entretanto, aos conteúdos, ao contexto e ao grupo social de reali- } \\
\text { zação do estudo. }\end{array}$ \\
\hline Estudo III & $\begin{array}{l}\text { Considerando as análises dos resultados, as revelações dos IPA e os indícios apresentados, con- } \\
\text { cluiu-se que o Estudo III não reuniu elementos que sinalizassem para a possibilidade de exis- } \\
\text { tência de uma possível relação de implicação dialógica entre os aprendizados dos conhecimen- } \\
\text { tos físicos e matemáticos. }\end{array}$ \\
\hline Estudo IV & $\begin{array}{l}\text { Os resultados do trabalho realizado no Estudo IV não apontam para ocorrência de uma relação } \\
\text { dialética de possíveis aprendizados entre os conteúdos da Matemática e da Física envolvidos no } \\
\text { processo instrucional. Entretanto, o ambiente proporcionado no contexto dos conteúdos da Fí- } \\
\text { sica pode ter contribuído com a assimilação dos assuntos da Matemática, uma vez que o estudo } \\
\text { dos primeiros favoreceu o uso constante das funções e equações lineares, conteúdos matemáti- } \\
\text { cos desta investigação. }\end{array}$ \\
\hline Estudo V & $\begin{array}{l}\text { As constatações e indícios nesta quinta e última etapa (Estudo V) do trabalho de investigação } \\
\text { apontam para a inexistência de possível relação dialética entre aprendizagens dos conteúdos da } \\
\text { Matemática e da Física envolvidos no processo instrucional. }\end{array}$ \\
\hline
\end{tabular}

Considerando a primeira premissa, pode-se dizer que algumas condições para a ocorrência de aprendizagem significativa podem não ter sido satisfatórias e que, por conseguinte, os resultados da investigação, possivelmente, não contribuíam substancialmente para responder o problema de pesquisa. Ou seja, respaldados em princípios norteadores da Teoria da Aprendizagem Significativa (AUSUBEL, 2002; MOREIRA, 2006), alguns fatores, tais como o material potencialmente significativo e a predisposição para aprender, aliados ao fator tempo para 
a aquisição e organização de significados, podem interferir na ocorrência da aprendizagem significativa.

Buscando melhor entender esses fatores, Moreira (2006), fundamentado na TAS, faz algumas considerações. Referindo-se ao material potencialmente significativo, ele diz:

\begin{abstract}
A condição de que o material seja potencialmente significativo envolve dois fatores principais, ou duas condições subjacentes, quais sejam, a natureza do material, em si, e a natureza da estrutura cognitiva do aprendiz. Quanto à natureza do material, ele deve ser "logicamente significativo" ou ter "significado lógico", isto é, ser suficientemente não arbitrário e não aleatório, de modo que possa ser relacionado, de forma substantiva e não arbitrária, a ideias correspondentemente relevantes, que se situem no domínio da capacidade humana de aprender. No que se refere à natureza da estrutura cognitiva do aprendiz, nela devem estar disponíveis os conceitos subsunçores específicos, com os quais o novo material é relacionável (p. 19).
\end{abstract}

Referente à predisposição para a ocorrência da aprendizagem significativa, ele assevera:

Essa condição implica que, independentemente de quão potencialmente possa ser o material a ser aprendido, se a intenção do aprendiz for, simplesmente, a de memorizá-lo arbitrária e literalmente, tanto o processo de aprendizagem como seu produto serão mecânicos (ou automático). E de modo recíproco, independentemente de quão disposto a aprender estiver o indivíduo, nem o processo nem o produto da aprendizagem serão significativos, se o material não for potencialmente significativo - se não for relacionável à estrutura cognitiva, de maneira não literal e não arbitrária (ibid., p. 20).

Enquanto detalhamos as condições para a ocorrência da aprendizagem significativa, nos colocamos também a pensar, obviamente, sobre a avaliação da mesma e esta, diferentemente do que ocorre na concepção comportamentalista e tradicional de avaliação, implica em avaliar a compreensão, a aquisição de significados, além da capacidade de transferir o conhecimento adquirido a situações novas e fora da rotina do indivíduo (Moreira, 2011). Entretanto, "[...] a aprendizagem é progressiva, grande parte do processo ocorre na zona cinza, na região do mais ou menos, na qual o erro é normal" (ibid., p. 52). Trata-se, portanto, de um processo que requer uma nova forma de avaliar, predominantemente formativa e recursiva, além de processual. Ou seja, uma avaliação que ocorra ao longo do próprio processo instrucional e oportunize ao sujeito aprendiz fazer e refazer recursivamente suas tarefas de aprendizagem, o que certamente demandará tempo. Isto foi previsto na elaboração da UDE e oportunizado ao longo da realização das intervenções didáticas.

Embora estivéssemos atentos quanto aos fatores essenciais para uma instrução com base na TAS, quando da elaboração da UDE e a sua implementação nos diversos estudos, fazse necessário ressaltar que o período de tempo dispensado para a realização de cada Estudo (aproximadamente cinquenta dias) pode ter sido um fator comprometedor, tendo em vista a 
expectativa para a ocorrência da aprendizagem significativa dos conteúdos estudados. Essa constatação leva-nos a admitir que, possivelmente, os resultados decorrentes de cada Estudo podem não ter sido suficientemente consistentes ao ponto de gerar possibilidades de conclusões seguras e maduras, na perspectiva de um processo educacional com base nos princípios instrucionais da TAS.

Estas considerações encontram reforço na Teoria dos Campos Conceituais de G. Vergnaud (VERGNAUD, 1993; 1996b; MOREIRA, 2004), na qual, para o autor, citado por Moreira (2004), o conhecimento se organiza em campos conceituais e seu domínio, por parte do sujeito, ocorre no decorrer de um longo intervalo de tempo. Ou seja,

O domínio de um campo conceitual, não ocorre em alguns meses, nem mesmo em alguns anos. Ao contrário novos problemas e novas propriedades devem ser estudados ao longo de vários anos se quisermos que os alunos progressivamente os dominem. De nada serve tentar contornar as dificuldades conceituais; elas são superadas na medida em que são encontradas e enfrentadas, mas isso não ocorre de um só golpe (VERGNAUD, apud MOREIRA, 2004, p. 8).

Nas palavras de Vergnaud, encontramos respaldo que pode justificar a qualidade dos resultados oriundos dos Estudos, uma vez que estes decorreram da implementação de uma UDE composta de um conjunto de intervenções didáticas, cujo processo educativo, em cada Estudo, visava proporcionar o aprendizado dos conteúdos da Física (quantidade de movimento e sua conservação) e da Matemática (funções e equações lineares), pertencentes aos complexos campos conceituais da Mecânica Clássica e da Álgebra, respectivamente.

Com relação à segunda premissa da questão de pesquisa, possibilidades de interferência dialógica entre os conteúdos matemáticos e físicos, pode-se encontrar alguma explicação na maneira como tais conhecimentos se definem um para o outro. Ou seja, como o conhecimento matemático é visto no contexto da Física e vice-versa. Dentre os estudos analisados para fins da Revisão de Literatura deste trabalho de pesquisa, destacamos alguns que, de alguma maneira, colaboraram para melhor entender esta relação dialógica e, consequentemente, contribuíram com elementos que nos possibilitaram inferir sobre possibilidades da real existência (ou não) de tal relação nesta investigação.

Comentando sobre o conhecimento físico e sua relação com a Matemática, Martini (2006), ao destacar a visão do físico-cientista e do aluno sobre esta relação, sentencia que a Matemática parece ser estruturante do próprio conhecimento para o físico-cientista, uma vez que não há qualquer acontecimento ou fenômeno que possa adquirir significado sem que a Matemática lhe atribua "realidade" e lhe legitime. Já para o aluno, ao contrário, o caráter estruturante da matemática é de outra categoria, pois é nela que o aluno encontra e, invariavelmente, reconhece o suporte para sua aprendizagem.

Redish (2005) chama a atenção para o fato de que apenas desenvolver habilidades de manipulação de fórmulas não é o suficiente no ensino de Física. É preciso melhorar a 
compreensão dos processos cognitivos envolvidos no problema de Física e encontrar atividades que ajudem os alunos a construirem conhecimentos em intuição e compreensão.

Concordando com este último autor, Sherin (2001) também coloca que apenas manipulação mecânica de expressões matemáticas não é o suficiente. Sugere que o uso de expressões simbólicas pode envolver a compreensão significativa e destaca o papel da modelagem matemática no estudo de situações da Física. Para ele, antes da instrução em Física, os alunos devem aprender a inventar pelo menos alguns tipos simples de modelos matemáticos e expressar o conteúdo destes modelos. E mais, que isto deve se constituir em um objetivo central da instrução em Física.

Por sua vez, Pietrocola (2002) destaca o papel estruturante da Matemática como linguagem do conhecimento físico, na medida em que considera que a Matemática é a maneira de estruturarmos nossas ideias sobre o mundo físico. Ele considera a realidade não como ponto de partida, mas de chegada das interpretações científicas e, neste processo, a Matemática, como linguagem, empresta sua própria estruturação ao pensamento científico para compor os modelos físicos sobre o mundo (ibid.). Ainda reportando-se sobre o poder estruturante da Matemática no ensino de Física, o autor chama a atenção dos educadores, quando diz:

Ao concebermos a apreensão do real como fruto de um processo de interação dialética entre o abstrato e concreto, entre o teórico e empírico, não há como evitar o tratamento da Matemática como elemento que participa, com sua especificidade própria, do contexto da construção do conhecimento. Assim, um dos atributos essenciais ao educador com relação a esta questão é perceber que não se trata apenas de saber matemática para poder operar as teorias físicas que representam a realidade, mas de saber aprender teoricamente o real através de uma estruturação matemática (ibid., p. 111).

Como se observa, no contexto dos trabalhos supracitados, o papel da Matemática no estudo do conhecimento físico requer alguns atributos, que certamente buscam romper com as crenças ingênuas oriundas do senso comum (Anjos, Caballero e Moreira, 2011). Seja na compreensão dos processos cognitivos envolvidos no problema da Física (Redish, 2005) ou na condição de atribuir "realidade" a um fenômeno físico (Martini, 2006), seja no aspecto da modelagem no estudo de situações Física ou ainda como estruturante do conhecimento físico (Pietrocola, 2002), a Matemática deve ser para a Física uma linguagem que ajuda a estruturar o pensamento científico.

Nesse sentido, como diz Pietrocola (2002), com base na sua própria experiência de professor de Física do Ensino Médio e Universitário,

[...] não basta ao aluno conhecer a Matemática no seu campo próprio de validade para obter um bom desempenho em Física. Isto é, não é suficiente conhecê-la enquanto "ferramenta" para utilizá-la como estruturante das ideias físicas sobre o mundo (p. 111). 
Em outras palavras, não se trata de saber Matemática como algo preliminar para compreender a Física. É preciso concebê-la como linguagem estruturante de apreensão da realidade física. Como diz ainda o próprio Pietrocola (2002):

Se a matemática é a linguagem que permite ao cientista estruturar seu pensamento para apreender o mundo, o ensino da ciência deve propiciar meios que os estudantes adquiram esta habilidade. Não parece que um mero domínio operacional dos conteúdos matemáticos seja capaz de permitir a incorporação de tal habilidade (p. 111).

Tendo em vista as considerações sobre as relações entre a Matemática e a Física, passemos a considerar os resultados e inferências da pesquisa, advindos de cada um dos Estudos e sinteticamente apresentados nos Quadros 2 e 3. À Exceção do Estudo I (Estudo piloto), que objetivou avaliar a UDE, os demais Estudos apresentaram indícios que vamos a analisar à luz das considerações emanadas de investigações na área e das teorias que dão suporte a este trabalho.

Os resultados oriundos do Estudo II apresentaram indícios cuja análise levou-nos a concluir (Quadro 2) que o Estudo II reúne elementos que, aparentemente, sinalizam para a existência de uma possível relação de implicação dialógica entre os aprendizados significativos dos conhecimentos físicos e matemáticos, muito embora se observe mais acentuadamente no sentido do aprendizado matemático no contexto da Física. Possivelmente, as oportunidades proporcionadas pela resolução de exercícios e situações problemáticas na Física parecem contribuir para o desenvolvimento de conhecimentos procedimentais dos conteúdos matemáticos.

Quanto aos demais Estudos (III, IV e V), quando comparados ao Estudo II, não apresentaram maiores discrepâncias entre si. Em todos eles, assim como também no próprio Estudo II, os indícios revelados (Quadro 2) sinalizam para o fato dos estudantes ao demonstrarem domínio sobre os conhecimentos da Matemática, não garantem evidências do reflexo disto no estudo dos conhecimentos físicos.

No Estudo III, por exemplo, os indícios detectados oferecem-nos poucas condições para se concluir sobre a existência e tipo de relação entre os saberes físicos e matemáticos, tampouco sinalizam para o efeito estruturante da Matemática numa situação física, tendo em vista que os resultados obtidos apresentam-se pouco consistentes para admitir tais constatações. Entretanto, considerando as análises dos resultados, as revelações dos Indicadores (IPA) e os indícios apresentados, concluiu-se que este Estudo III não reuniu elementos que sinalizem para a possibilidade de existência de uma possível relação de implicação dialógica entre aprendizados significativos dos conhecimentos físicos e matemáticos.

Não muito diferente, o Estudo IV também apresentou resultados cuja análise nos conduziu a concluir que $o$ trabalho realizado nesta etapa da investigação não aponta para ocorrência de uma relação dialética entre possíveis aprendizados dos conteúdos da Matemática e da Física envolvidos no processo de instrução didático-pedagógica. Entretanto, o ambiente proporcionado no contexto dos conteúdos da Física pode ter contribuído para a assimilação 
dos assuntos da Matemática, uma vez que o estudo dos primeiros favoreceu o uso constante das funções e equações lineares, conteúdos matemáticos desta investigação.

No quinto e último estudo (Estudo V), os indícios constatados nesta etapa da investigação levaram-nos a inferir que a pesquisa realizada aponta para a inexistência de possível relação dialética entre as aprendizagens dos conteúdos matemáticos e físicos envolvidos no processo instrucional, embora também revele uma forte tendência do contexto da Física ter contribuído para a aprendizagem dos conteúdos da Matemática.

\section{Conclusão}

Considerando as revelações já apresentadas, decorrentes dos resultados obtidos com esta pesquisa, devidamente tratados e analisados à luz dos aportes teóricos que dão suporte a este trabalho de investigação, constatam-se algumas situações que se repetem, parcial ou integralmente, em todos ou quase todos os Estudos. São situações que pelo aspecto repetitivo nos Estudos revestem-se da condição de regularidade e, como tal, certamente contribuíram substancialmente com elementos para as considerações conclusivas da nossa pesquisa.

A primeira regularidade, verificada em todos os Estudos, diz respeito ao domínio conceitual e operacional das equações lineares. Constatou-se, através das observações e registros (avaliação processual) ao longo do desenvolvimento das intervenções didáticas e ao final com o TAF que os estudantes responderam relativamente bem no que se refere a este conhecimento.

O entendimento do conteúdo função linear constituiu-se na segunda regularidade. Exceto no Estudo IV, no qual o domínio deste conhecimento não foi satisfatório, nos demais casos verificou-se progressão na aprendizagem deste conteúdo, tendo em vista o nível de conhecimento inicialmente apresentado pelos estudantes, sua evolução ao longo do processo educativo e as condições apontadas pelos IPA.

A compreensão do conceito de quantidade de movimento, apenas no aspecto da formalização matemática, revelou-se como a terceira regularidade. Ficou constatado em todos os Estudos que o domínio deste conceito sempre ocorrera sob o ponto de vista funcional. Isto é, para um corpo de massa (m) considerada constante, o valor da quantidade de movimento $(\mathbf{Q})$ é proporcional ao valor da velocidade $(\mathbf{v})$.

A quarta regularidade manifestada em quase todos os casos, refere-se à não compreensão, uso e aplicação do princípio de conservação da quantidade de movimento. Isto se verificou e ficou bastante evidenciado quando os alunos eram solicitados a resolverem situações problemáticas envolvendo interações entre corpos. Mesmo quando se conseguia encontrar uma solução para o problema, via-de-regra, esta não vinha acompanhada do uso adequado do princípio, tanto no aspecto matemático como no físico. Foram constatações observadas pelo professor ao mediar o processo instrucional nas intervenções didáticas e, também, confirmadas nas Entrevistas, nas quais os estudantes foram solicitados a explicitarem os seus conhecimentos 
utilizados nas propostas de resolução das questões. Mesmo no Estudo II, onde houvera considerada progressividade no trato com problemas do tipo, o uso do princípio restringiu-se ao aspecto matemático.

Como já é conhecido, este trabalho de pesquisa objetivou investigar a relação dialética entre as aprendizagens significativas de conteúdos da Matemática (funções e equações matemáticas) e da Física (quantidade de movimento e sua conservação). Coerente com os objetivos da pesquisa tentou-se ao longo da investigação encontrar evidências e regularidades, cujos elementos constituintes, de alguma forma, colaboraram para elucidar a problemática de investigação do trabalho.

Esses achados (evidências e regularidades) levaram-nos a conclusões, as quais podem ser consideradas como possibilidades de respostas à pergunta de pesquisa e que, visando melhor entendimento, serão explicitados considerando, inicialmente, as aprendizagens dos conhecimentos físicos e matemáticos distintamente e, posteriormente, a relação dialógica entre esses aprendizados.

Quanto às possibilidades de aprendizagem significativa nos campos da Matemática e da Física, os achados nos conduziram a inferir que, mesmo com a inadequação do tempo para possibilidades de ocorrência de tal aprendizagem, até onde se pode perceber, é provável que, no âmbito da Matemática, o trabalho didático-pedagógico tenha favorecido tal ocorrência e que provavelmente isto se deva em decorrência das intervenções didáticas. Já no campo da Física, o mesmo não se pode afirmar, ou seja, a aquisição do conhecimento acerca do princípio de conservação da quantidade de movimento possivelmente não ocorrera e, quando aparentemente se evidenciara (Estudo II), mostrara-se com consistência nos aspectos matemáticos e pouco consistente no âmbito da Física, sobretudo no aspecto do tratamento vetorial das grandezas (quantidade de movimento e velocidade) nas equações.

No que se refere à relação de interferência dialógica entre possíveis aprendizados dos conteúdos matemáticos e físicos, os achados nos levaram a concluir que não são suficientemente consistentes para se ter um parecer abalizado e convincente sobre tal interferência, respaldado em considerações de cunhos racional e lógico, a saber: racional, uma vez que razões, como por exemplo, pouco tempo para o processo educativo e complexidade dos campos conceituais e outras já comentadas, não são substanciais para se falar em aprendizagem significativa; lógico, porque não se tendo convicção de aprendizagem dos citados conteúdos pelas razões explicitadas, logicamente, não se pode falar de possível interferência recíproca de aprendizados.

Entretanto, vale assinalar, como já fora mencionado, que existem fortes indícios de que os processos de ensino e aprendizagem dos conhecimentos físicos tenham-se constituído num terreno fértil e propício para a aprendizagem dos conhecimentos matemáticos e, dessa forma, podendo favorecer a aprendizagem significativa destes conhecimentos.

Analisando mais uma vez a questão e buscando ser fiel e coerente com ela, diríamos que, embora a investigação tenha proporcionado bons resultados, muitos indícios e evidências, 
constata-se que estes elementos não foram suficientemente consistentes para responder na íntegra o questionamento da pesquisa.

\section{Referências}

ALVARADO, M. S. A. Dificultades en la aplicación del cálculo diferencial e integral en la resolución de problemas del trabajo efectuado por un gas ideal: Conceptos y teoremas en acción. Latin American Journal of Physics Education, v. 6, n. 1, p. 106-115, 2012.

ANJOS, A. J. S. El aprendizaje en Física bajo el punto de vista del significado atribuido por los estudiantes a las ecuaciones matemáticas. 2009. 99f. Suficiencia de Investigación (Programa Internacional de Doctorado en Enseñanza de las Ciencias) - Universidad de Burgos, Burgos, España.

ANJOS, A. J. S.; CABALLERO, M. C.; MOREIRA, M. A. A aprendizagem em Física sob o ponto de vista do significado atribuído pelos estudantes às equações matemáticas. In: ENCUENTRO IBEROAMERICANO SOBRE INVESTIGACIÓN EN ENSEÑANZA DE LAS CIENCIAS, III, 2009, Burgos, Espanha. Atas... Burgos: Universidad de Burgos, 2005.

ARIZA, A.; LINARES, S. Sobre la aplicación y uso del concepto de derivada en el estudio de conceptos económicos en estudiantes. Enseñanza de las Ciencias, Barcelona, Espanha, v. 27, n.1, p. 121-136, 2009.

ATAÍDE, A. R. P.; GRECA, I. M. Estudo exploratório sobre as relações entre conhecimento conceitual, domínio de técnicas matemáticas e resolução de problemas em estudantes de licenciatura em Física. Revista Eletrónica de Enseñanza de las Ciencias, v.12, n.1, p. 209-233, 2013.

AUSUBEL, D. Aquisição e retenção de conhecimentos: Uma perspectiva cognitiva. Lisboa: Editora Plátano, 2003.

AYUSH, G.; REDISH, E. F.; HAMMER, D. In: COORDINATION OF MATHEMATICS AND PHYSICAL RESOURCES BY PHYSICS GRADUATE STUDENTS. AIP Conference Proceedings... v. 951, p. 104-107, 2007.

BAGNO, E.; EYLON, B.; BERGER, H. How to promote the learning of Physics from formulae? GIREP-EPEC 2009 Symposium, 2009.

DOMERT, D.; AIREY, J.; KUNG, R.; LINDER, C. An exploration of university physics students' epistemological mindsets towards the understanding of physics equations. NorDiNaNordic Studies in Science Education, v. 3. n. 1, p. 15-28, 2007.

GALAGOUSKY, L. R.; CITTADINI, P. E. Enseñanza de equaciones lineales en contexto. Enseñanza de las Cienciais, v. 26, n. 3, p. 359-374, 2008. 
HECHTER, R. P. What does 'i understand the equation' really mean? Physics Education, v. 45, n. 2, p. 132-133, 2010.

KARAM, R. Grandezas físicas para exemplificar a Função Afim. Revista do Professor de Matemática, Rio de Janeiro: Sociedade Brasileira de Matemática, n. 63, p. 29-35, 2007.

KARAM, R.; PIETROCOLA, M. Habilidades técnicas versus habilidade estruturantes: resolução de problemas e o papel da Matemática como estruturante do pensamento científico. ALEXANDRIA Revista de Educação em Ciência e Tecnologia, v. 2, n. 2, p. 181-205, 2009.

KARAM, R.; PIETROCOLA, M. Recognizing the structural role of Mathematics in physical thought. In: EUROPEAN SCIENCE EDUCATION RESEARCH ASSOCIATION CONFERENCE, 2009, Istanbul, Turkey. p. 1-10.

KARAM, R. Grandezas físicas para exemplificar a função afim. 2004. Disponível em: <http://www.nupic.fe.usp.br>. Acesso em: 18 jul. 2013.

LOPES, J. P. Fragmentações e Aproximações entre Matemática e Física no Contexto Escolar: problematizando conceito de função afim. 2004. 180 f. Dissertação (Mestrado em Ensino de Ciências) - Universidade Federal de Santa Catarina, Florianópolis.

LÓPEZ-GAY, R.; MARTÍNEZ-TORREGROSA, J. Análisis de la utilización y comprensión del Cálculo Diferencial en la enseñanza de la Física. Enseñanza de las Cienciais, v. 23, n. 3, p. 321-334, 2005.

LÓPEZ-GAY, R.; MARTINEZ-TORREGROSA, J. Que hácen y qué entienden los estudiantes y profesores de Física cuando usan expressiones diferenciales? Enseñanza de las Cienciais, v, 23, n. 3, p. 331-334, 2005.

LUDKE, M.; ANDRÉ, M. E. D. A. Pesquisa em Educação: abordagens qualitativas. São Paulo: EPU, 2003.

MARTINI, M. da G. de A. O conhecimento físico e sua relação com a Matemática: um olhar voltado para o ensino médio. 2006. 151 f. Dissertação (Mestrado em Ensino de Física) - Universidade de São Paulo, São Paulo.

MARTINEZ-TORREGROSA, J.; LOPEZ-GAY, R.; GRAS-MARTI, A. Mathematics in Physics Education: scaning historical evolution of the differencial to find a more appropriate model for teaching Differencial Calculus in Physics. Science and Education, v. 15, n. 5, p. 447-462, 2006.

MOREIRA, M. A. A Teoria dos Campos Conceituais de Vergnaud, o ensino de ciências e a investigação nesta área. Porto Alegre: I.F. da UFRGS, 2004. 
MOREIRA, M. A. A teoria da aprendizagem significativa e sua implementação em sala de aula. Brasília: Editora da Universidade de Brasília, 2006.

MOREIRA, M. A. Aprendizagem significativa: a teoria e textos complementares. São Paulo: Editora Livraria da Física, 2011.

MOREIRA, M. A.; KREY, I. Dificuldades dos alunos na aprendizagem da lei de Gauss em nível de Física Geral à luz da teoria dos modelos mentais de Johnson-Laird. Revista Brasileira de Ensino de Física. São Paulo, v. 28, n. 3, p. 353-360, 2006.

MOREIRA, M. A.; PINTO, A. O. Dificuldades dos alunos na aprendizagem da Lei de Ampére, à luz da Teoria dos Modelos Mentais de Johnson-Laird. Revista Brasileira de Ensino de Física, v. 25, n. 3, p. 317-324, 2003.

MULHALL, P.; GUNSTONE, R. Views about Physics held by Physics teachers with differing approaches to teaching Physics. Research Science Education, v. 38, p. 435-462, 2008.

OLIVEIRA, A. T. de S. Fórmulas e palavras: reflexões sobre o ensino da Lei de Coulomb. 2003. 135 f (Tese de Doutorado) - Universidade Federal Fluminense, Rio de Janeiro.

ORNEK, F.; ROBINSON, W. R.; MARK P. HAUGAN, M. P. What makes physics difficult? International Journal of Environmental \& Science Education, v. 3, n. 1, p. 30-34, 2008.

ORTON, T.; ROPER, T. Science and Mathematics: a relationship in need of counseling? Studies in Science Education, v. 35, p. 123-154, 2000.

PIETROCOLA, M. A Matemática como estruturante do conhecimento físico. Caderno Brasileiro de Ensino de Física, v. 19, n. 1, p. 93-114, 2002.

QUALE, A. On the Role of Mathematics in Physics: A Constructivist Epistemic Perspective. Science \& Education, v. 20, p. 609-624, 2011.

QUALE, A. On the Role of Mathematics in Physics. Science \& Education, v. 20, p. 359-372, 2011.

REDISH, E. F. Problem Solving and the use of Math in Physics Courses. World View on Physics Education in 2005: Focsing on Change, Delhi, p. 1-10, 2005.

REDISH, E. F. Solving and the use of Math in Physics courses. Invited talk presented the conference, Word View on Physics Education in: focusing on change. Delhi, 2005.

REDISH, E. Using Math in Physics: Warrants an Epistemological Frames. In: GIREP-EPEC Symposium, 2009.

SANTAROSA, M. C. P.; MOREIRA, M. A. O Cálculo nas aulas de Física da UFRGS: um estudo exploratório. Investigações em Ensino de Ciências, v. 16, n. 2, p. 317-351, 2011. 
SHERIN, B. L. How students understand physics equations. Cognition and Instruction. Lawrence Erlbaum Associates Inc. v, 19, n. 4, p. 479-541, 2001.

VERGNAUD, G. A teoria dos campos conceituais. In: NASSER, L. (Ed.). SEMINÁRIO INTERNACIONAL DE EDUCAÇÃO MATEMÁTICA, 1993, Rio de Janeiro. Anais... p. 1-26.

ZUFFI, E. M.; PACCA, J. L. de A. O conceito de função e sua linguagem para os professores de Matemática e de Ciências. Ciência e Educação, v. 8, n. 1, p. 1-12, 2002. 